\title{
Prognostic Impact of Pelvic Lymph Node Dissection During Radical Prostatectomy on Patients With High-risk Prostate Cancer Treated With Neoadjuvant Chemohormonal Therapy
}

Hiromichi Iwamura

Hirosaki University Graduate School of Medicine

Shingo Hatakeyama ( $\square$ shingoh@hirosaki-u.ac.jp)

Hirosaki University Graduate School of Medicine

Takuma Narita

Hirosaki University Graduate School of Medicine

Yusuke Ozaki

Hirosaki University Graduate School of Medicine

Sakae Konishi

Hirosaki University Graduate School of Medicine

Hirotaka Horiguchi

Hirosaki University Graduate School of Medicine

Hirotake Kodama

Hirosaki University Graduate School of Medicine

Yuta Kojima

Hirosaki University Graduate School of Medicine

Naoki Fujita

Hirosaki University Graduate School of Medicine

Teppei Okamoto

Hirosaki University Graduate School of Medicine

Yuki Tobisawa

Hirosaki University Graduate School of Medicine

Tohru Yoneyama

Hirosaki University Graduate School of Medicine Hayato Yamamoto

Hirosaki University Graduate School of Medicine

Takahiro Yoneyama

Hirosaki University Graduate School of Medicine

Yasuhiro Hashimoto

Hirosaki University Graduate School of Medicine 


\section{Chikara Ohyama}

Hirosaki University Graduate School of Medicine

\section{Research Article}

Keywords: prostate cancer, prognosis, lymph node dissection, neoadjuvant chemohormonal therapy, inverse probability of treatment weighting

Posted Date: December 22nd, 2021

DOI: https://doi.org/10.21203/rs.3.rs-1161305/v1

License: (c) (1) This work is licensed under a Creative Commons Attribution 4.0 International License. Read Full License 


\section{Abstract}

\section{Background}

We aimed to determine the prognostic and staging benefit of limited pelvic lymph node dissection (PLND) during radical prostatectomy (RP) in high-risk prostate cancer (PC) patients treated with neoadjuvant chemohormonal therapy.

\section{Methods}

We retrospectively analyzed 516 patients with high-risk localized PC (<CT4NOM0) who received neoadjuvant androgen-deprivation therapy plus estramustine phosphate followed by RP between January 2010 and March 2020. Since we stopped limited-PLND for such patients in October 2015, we compared the biochemical recurrence-free survival (BCR-FS) between the limited-PLND group (before October 2015, $n=283$ ) and the non-PLND group (after November 2015, $n=233$ ).

\section{Results}

The rate of node metastases in the limited-PLND group were $0.8 \%(2 / 283)$. Operation time was significantly longer (176 vs. $162 \mathrm{~min}$ ) and the rate of surgical complications were much higher (all grades; 19 vs. $6 \%$, grade $\geq 3 ; 3$ vs. $0 \%$ ) in the limited-PLND group. The inverse probability of treatment weighting-Cox analysis revealed limited PLND had no significant impact on BCR-FS (hazard ratio, 1.31; P $=0.421)$.

\section{Conclusions}

Limited PLND during RP after neoadjuvant chemohormonal therapy showed a relatively low rate of positive nodes, higher rate of complications, and no significant impact on BCR-FS.

\section{Introduction}

Pelvic lymph node dissection (PLND) during radical prostatectomy (RP) for localized prostate cancer (PC) has been performed since the procedure was established in the 1980s. ${ }^{1}$ Although PLND can accurately determine the nodal stage, its indications, optimal extent, and therapeutic benefits remain controversial because of the lack of randomized controlled trials (RCTs). ${ }^{2}$

Previous retrospective studies suggested that PLND during RP may have survival benefits mainly because some patients with pathological proven lymph node metastasis did not relapse without adjuvant therapy. ${ }^{3-7}$ Recently, two RCTs comparing the extended and the limited PLND have been reported. The results showed that extended PLND did not improve the biochemical recurrence-free survival (BCR-FS) compared with limited PLND. ${ }^{8,9}$ These results indicated that there is no difference in prognosis based on the extent of PLND. However, RCTs comparing PLND from no PLND remain unavailable. Furthermore, 
there are no reports, including retrospective studies, evaluating the oncological and staging benefits of PLND during RP in patients with high-risk PC who received neoadjuvant therapy.

In our institution, all patients with high-risk PC undergoing RP have been treated with neoadjuvant androgen-deprivation therapy (ADT) plus low-dose estramustine phosphate (EMP) and limited PLND. ${ }^{10,11}$ Because the rate of positive nodes in those patients was less than $1 \%$, we questioned the significance of limited PLND and stopped limited PLND during RP in October 2015. Thus, the aim of the present study was to assess the oncological benefits of limited PLND during RP on BCR-FS in patients with high-risk PC who received neoadjuvant ADT plus low-dose EMP by comparing the outcomes before and after the discontinuation of PLND.

\section{Results}

\section{Baseline characteristics}

We identified 574 patients with high-risk localized PC (<cT4NOM0) who have received neoadjuvant therapy followed by RP between January 2010 and March 2020. Of those, we excluded patients treated with other neoadjuvant regimens $(n=41)$ and those who discontinued low-dose EMP during the neoadjuvant period due to adverse events $(n=17)$. Finally, we included 516 patients with high-risk PC treated with neoadjuvant chemohormonal therapy (ADT plus low-dose EMP) followed by RP. Among them, 283 underwent limited PLND and 233 did not. The limited-PLND group had a significantly higher rate of biopsy International Society of Urological Pathology grade group $5(56.2 \%$ vs. $45.5 \%, P=0.022)$ and a significantly lower rate of cT3 than the non-PLND group (30.7\% vs. $53.2 \%, P=0.001)$. The median risk of lymph node invasion was $16 \%$. The median duration of the neoadjuvant chemohormonal therapy was 8.3 months (Table 1 ).

\section{Surgical and pathological outcomes}

Considering the historical cohort study, the surgery type was significantly different between the groups; the rate of robot-assisted radical prostatectomy (RARP) was $53.7 \%$ and $97.4 \%$ in the limited-PLND and non-PLND groups, respectively $(P<0.001)$. Accordingly, the non-PLND group had a significantly longer median operation time (152 vs. $161 \mathrm{~min}, \mathrm{P}=0.001$ ) and significantly lesser median blood loss (100 vs. $25 \mathrm{~mL}, \mathrm{P}<0.001$ ) than the other group (Table 2). In patients who received RARP, the operation time was significantly shorter in the non-PLND group than in the limited-PLND group (176 vs. $162 \mathrm{~min}, \mathrm{P}=0.001$ ), but the blood loss was not significantly different between the groups ( $30 \mathrm{vs} .25 \mathrm{~mL}, \mathrm{P}=0.593$ ). The incidence of surgical complications was significantly higher (all grades; 19 vs. $6 \%, P<0.001$, grade $\geq 3 ; 3$ vs. $0 \%, P=0.010$ ) in the limited-PLND group (Fig. 1a-d, Table S1). The pathological tumor stage was not significantly different between the groups, but the rate of surgical margin positivity was significantly higher in the non-PLND group than in the limited-PLND group $(6.0 \%$ vs. $11.6 \%, P=0.027)$ (Table 2).

In the limited-PLND group, the median number of dissected nodes was 4 (interquartile range: $3-7$ ) and the rate of positive nodes was $0.8 \%$ (2/283) (Table 2). Age, anticoagulant use, surgery type, clinical tumor 
stage, and biopsy Gleason score had no significant impact on the number of dissected nodes (Fig. 2a-e, Table S2).

\section{Oncological outcomes}

The median follow-up period was significantly longer in the limited-PLND group than in the non-PLND group (77 vs. 34 months, $P<0.001$ ). Accordingly, BCR was observed in 55 and 30 patients in the limitedPLND and non-PLND groups, respectively (Table 2). The unadjusted Kaplan-Meier analysis showed that BCR-FS was not significantly different between the groups (3 years, $89.1 \%$ vs. $86.0 \%$; 5 years, $84.1 \%$ vs. $82.0 \% ; P=0.516$, Fig. 3a). According to the multivariate Cox regression analysis, age, initial prostatespecific antigen (PSA), pathological tumor stage, and surgical margin were independent prognostic factors for BCR-FS (HR: 0.95, 1.02, 2.49, and 1.94, respectively), whereas PLND was not (Table 3). Furthermore, the inverse probability of treatment weighting (IPTW)-adjusted Cox regression analysis, in which the potential confounders for BCR-FS (age, initial PSA, biopsy Gleason score, surgery type, pathological tumor stage, and surgical margin) were adjusted in both groups, showed that BCR-FS was not significantly different between the two groups (HR: 1.31 ; $=0.421$, Fig. $\mathbf{3 b}$ ).

\section{Discussion}

In this study, we evaluated the oncological benefits of limited PLND during RP in patients with high-risk PC treated with neoadjuvant ADT plus low-dose EMP, with adjustment for patients' background, surgery type, and clinical and pathological disease status using IPTW-adjusted model. Compared with no PLND, limited PLND had no significant impact on BCR-FS. Furthermore, PLND was significantly associated with a longer operation time and higher rate of surgical complications, with only $0.8 \%$ positive nodes.

The therapeutic benefit of PLND for malignancies has been debated in various types of cancer. Except colorectal cancers, ${ }^{12}$ bladder, ${ }^{13}$ esophageal, ${ }^{14}$ gastric, ${ }^{15}$ pancreatic, ${ }^{16}$ lung, ${ }^{17}$ breast, ${ }^{18}$ and ovarian cancers did not benefit from PLND in RCTs. ${ }^{19}$ Regarding PC, the therapeutic benefit of PLND during RP had been discussed according to the results of retrospective studies. Previous retrospective studies demonstrated that PLND during RP is a staging procedure and may also have a positive impact on survival. ${ }^{3-7}$ The main rationale for the prognostic benefit of PLND is that $24 \%$ of patients with positive lymph nodes did not relapse without adjuvant therapy ${ }^{3}$ and $30 \%$ of patients who underwent salvage LND for BCR and nodal recurrence had an undetectable PSA $(<0.1 \mathrm{ng} / \mathrm{mL}) .{ }^{4}$ Other studies argued that more extended PLND identified twice as many lymph node metastases as limited PLND, which may help to cure lymph node micrometastases. ${ }^{5-7}$ The 2021 EAU guidelines strongly recommend extended PLND for intermediate-risk PC with an estimated risk for lymph node metastases of $\geq 5 \%$ and all high-risk PC for optimal nodal staging. ${ }^{20}$ However, the 2017 AUA guidelines did not mention any recommendation because the therapeutic benefits of extended PLND still have no supporting evidence. ${ }^{21} \mathrm{~A}$ recent systematic review ${ }^{22}$ including 44 retrospective studies $(n=275,269)$ found that BCR-FS, metastatic-free survival, and cancer-specific survival were not significantly different between patients with PLND of any 
forms and those without PLND. Conversely, the more extensive the extent of PLND, the greater the adverse outcomes such as operating time, blood loss, postoperative complications, and hospitalization duration. ${ }^{22}$ Furthermore, two recently published RCTs demonstrated that extended PLND had no survival benefits compared with limited PLND (HRs for BCR-FS: 0.91 and 1.04, respectively). ${ }^{8,9}$ Although these two RCTs revealed that the extent of PLND has no impact on survival outcomes, RCTs comparing PLND and no PLND, particularly in patients treated with intensive neoadjuvant chemohormonal therapy, remain unavailable. In the present study, limited PLND during RP after neoadjuvant chemohormonal therapy showed no significant impact on survival outcomes, with a significantly longer operation time and higher rate of postoperative adverse events than no PLND. Furthermore, only two patients $(0.8 \%)$ had positive nodes, possibly because of the neoadjuvant chemohormonal therapy. Of the two patients, one had no BCR and the other had a PSA level of $>0.2 \mathrm{ng} / \mathrm{mL}$ immediately after surgery and died of PC 6 years later. Thus, of the 283 patients who underwent limited PLND, only one ( $0.4 \%$ ) had a therapeutic benefit from PLND and the others (99.6\%) underwent unnecessary PLND. These results suggest that limited PLND during RP may be omitted in patients with high-risk PC who have received neoadjuvant chemohormonal therapy. An ongoing trial comparing extended PLND and no PLND (NCT03921996) will help us elucidate whether PLND during RP can really be omitted, although there are no ongoing trials to determine whether neoadjuvant therapy can eliminate PLND.

Generally, PLND during RP is performed in $>90 \%$ of patients with high-risk $\mathrm{PC},{ }^{23}$ and the rate of positive nodes is $5 \%-37 \%$ in patients without neoadjuvant therapy. ${ }^{24-28}$ In a neoadjuvant setting, the rate of positive nodes is $0 \%-30 \%$, with various regimens of neoadjuvant therapy. ${ }^{29}$ Although guidelines do not recommend any neoadjuvant therapies outside clinical trials for patients who have elected to undergo $\mathrm{RP}^{20,21}$ neoadjuvant therapy is associated with a decreased rate of $\mathrm{pT} 3$, a reduced rate of positive surgical margin, and a lower incidence of lymph node metastases. ${ }^{30}$ In a recent meta-analysis, only regimens of neoadjuvant therapy comprising EMP significantly reduced the rate of positive nodes (odds ratio: $0.05-0.11) .{ }^{10,11,24,29}$ Similarly, the rate of positive nodes in the present study was only $0.8 \%(2 / 283)$ despite the relatively high estimated risk of lymph node invasion (median, 16\%). These results suggest that PLND is not beneficial even for nodal staging in patients with high-risk PC who received neoadjuvant chemohormonal therapy containing EMP. Nevertheless, selecting the optimal candidate for PLND remains necessary because some patients may still benefit from PLND with or without neoadjuvant therapy. The Briganti nomogram is the most common tool used to determine the indication for PLND during RP. ${ }^{31}$ With a $5 \%$ nomogram cutoff, $66 \%$ of patients will be spared from PLND. However, even with this nomogram, $27 \%$ of patients will still undergo unnecessary PLND (patients with a nomogram-derived lymph node metastasis risk of $\geq 5 \%$ without histologic lymph node invasion). Additionally, nomogram cannot be applied in the neoadjuvant setting. Meanwhile, novel imaging modalities such as whole-body magnetic resonance imaging ${ }^{32,33}$ and prostate-specific membrane antigen-targeted positron emission tomography (PSMA-PET) ${ }^{34,35}$ can differentiate patients with $\mathrm{cN} 1$ from those diagnosed with $\mathrm{cN} 0$ by conventional imaging. Ongoing trials (NCT04832958 and NCT04457245) for image-guided treatment protocols for localized PC using PSMA-PET will provide us with a novel treatment strategy for PLND during RP. 
Considering that surgery is complex and surgeon-dependent, some biases in surgical research need to be considered, even in RCTs. ${ }^{36}$ Surgeons may change the indication and extent of PLND according to the patients' background, disease status, and surgery type (selection and performance biases). Although this is a retrospective study with various surgeons, selection bias was minimized because the indication of PLND relied on the treatment strategy in our institution and not on the surgeons (all patients with highrisk PC underwent limited PLND before October 2015 and did not after November 2015). The IPTW analysis also contributed to minimizing the selection bias. Furthermore, all patients in the limited PLND group underwent the same lymphadenectomy technique. The number of lymph nodes dissected was not affected by patients' age, anticoagulant use, surgery type, clinical tumor stage, and biopsy Gleason score. Therefore, the present study has minimal selection bias and performance bias despite its retrospective design. To evaluate external validity, a multicenter retrospective study including our affiliated hospitals that follow the same treatment strategy as ours for PLND is warranted.

This study has some limitations. First, retrospective design and the limited sample size prevented us from attaining a definitive conclusion. Second, it is unclear whether the same results would be obtained with docetaxel or novel anti-androgens; the use of these drugs may further reduce the significance of lymph node dissection. Third, the follow-up period was significantly shorter in the non-PLND group because of the historical cohort design. Finally, our data cannot deny the benefits of extended PLND after neoadjuvant chemohormonal therapy. Despite these limitations, our study is the first to reveal that limited PLND during RP after neoadjuvant chemohormonal therapy does not improve BCR-FS in high-risk PC patients. Further research is needed to validate the present observation.

\section{Conclusion}

Limited PLND after neoadjuvant chemohormonal therapy containing EMP showed no significant impact on BCR-FS, with a relatively low rate of positive nodes. Thus, limited PLND during RP may be omitted in patients with high-risk PC who have received neoadjuvant chemohormonal therapy.

\section{Methods}

\section{Study design and participants}

We retrospectively analyzed consecutive patients with high-risk localized PC (<cT4NOM0) treated with neoadjuvant ADT plus low-dose EMP followed by RP at Hirosaki University Hospital between January 2010 and March 2020. We collected information regarding patients' background, disease status, and surgical, pathological, and oncological outcomes from their medical records. Patients with any missing information were excluded. We determined the tumor stage using the 2017 American Joint Committee on Cancer Staging Manual. ${ }^{37}$ We defined high-risk PC as follows: initial PSA levels of $\geq 20 \mathrm{ng} / \mathrm{mL}$, a biopsy Gleason score of $\geq 8$ (International Society of Urological Pathology grade group $\geq 4$ ), and/or clinical tumor stage T2c or T3 according to the D' Amico risk stratification. ${ }^{38}$ 


\section{Treatment procedures}

For 6-9 months before RP, all patients with high-risk PC who have elected to undergo RP received neoadjuvant ADT (luteinizing hormone-releasing hormone agonist or gonadotropin-releasing hormone antagonist) plus low-dose EMP (280 mg/day) in our institution, as previously described. ${ }^{10,11,24}$ With the approval of RARP in Japan in January 2012, RARP has been performed in most cases; in earlier cases, retropubic radical prostatectomy was performed. Considering that the rate of positive lymph nodes was approximately $1 \%$ in patients with high-risk PC treated with neoadjuvant ADT plus low-dose EMP followed by RP at our institution, ${ }^{24}$ we stopped PLND in October 2015. Thus, we categorized patients into two groups according to treatment strategy shift: limited-PLND group (patients with high-risk PC who received limited PLND; between January 2010 and October 2015) and non-PLND group (patients who did not receive limited PLND; after November 2015). The limited-PLND group underwent the same lymphadenectomy method, including removal of the bilateral obturator nodes. Surgical complications were evaluated using the Clavien-Dindo classification. ${ }^{39}$ The risk of lymph node invasion was retrospectively calculated using the Briganti nomogram. ${ }^{31}$

\section{Pathological analysis}

All prostatectomy specimens were sectioned using the whole-mount technique and then evaluated according to the International Society of Urological Pathology 2005/2014 guidelines. ${ }^{40,41}$ Each dissected lymph node was cut into 3-mm slices, embedded separately in paraffin, stained with hematoxylin and eosin, and examined under the microscope. The results were described as the total number of lymph nodes dissected and the number of positive lymph nodes. An expert urological pathologist reviewed all biopsy and surgical specimens.

\section{Follow-up}

Postoperatively, serum PSA and testosterone levels were evaluated in all patients every 3 months. These patients did not undergo adjuvant ADT or radiation therapy in our institution regardless of the pathological results. We defined the date of BCR as the date when the serum PSA level exceeded 0.2 $\mathrm{ng} / \mathrm{mL}$. If the PSA level did not decrease to less than $0.2 \mathrm{ng} / \mathrm{mL}$ postoperatively, the date of RP was considered as the date of BCR.

\section{Endpoints}

The primary endpoint was evaluating the therapeutic effect of limited PLND on BCR-FS. The secondary endpoints were comparing surgical outcomes and complications between the groups, and the benefit of limited PLND in terms of nodal staging.

\section{Statistical analysis}


All data were analyzed using R 4.0.2 (The R Foundation for Statistical Computing, Vienna, Austria) and GraphPad Prism 9.00 (GraphPad Software, San Diego, CA, USA). We compared categorical variables using the Fisher's exact test or $\chi^{2}$ test. Quantitative variables were expressed as median with an interquartile range. The student's $t$-test or the Mann-Whitney Utest was used to compare differences between groups. Using the Kaplan-Meier method, we compared the BCR-FS between the limited-PLND and non-PLND groups. Hazard ratios (HR) with 95\% confidence intervals (Cls) were obtained by multivariate Cox regression analysis using the IPTW-adjusted model. After creating a pseudopopulation, we used the propensity score-based IPTW method to remove the background imbalances between the groups and estimate the average treatment effect in an unbiased manner. ${ }^{42}$ Multivariate IPTW-Cox analysis included the following steps. First, we calculated the propensity score using the parameters including age, initial PSA, biopsy Gleason score, surgery type, pathological tumor stage, and surgical margin, for the limited-PLND group through logistic regression analysis. Second, we calculated the IPTW by the inverse probability of the "given" exposure. The treatment weights for the limited-PLND and nonPLND groups were determined using the following formula: $1 /$ propensity score and $1 /(1$ - propensity score), respectively. Third, we performed multivariable Cox regression analysis with robust adjustment including two factors: treatment selection (limited-PLND group $=1$ ) and treatment weighting. P-values of $<0.05$ were considered statistically significant.

\section{Declarations}

\section{Ethics approval statement}

This retrospective study was approved by the Ethics Committee of Hirosaki University (2019-099). All participants had previously provided written informed consent for other clinical studies. Since all the data used in this study were obtained from the medical records, additional informed consent for this study was waived with approval by the Ethics Committee of Hirosaki University. The clinical and research activities being reported are consistent with the Principles of the Declaration of Helsinki.

\section{References}

1. Hyndman, M. E., Mullins, J. K. \& Pavlovich, C. P. Pelvic node dissection in prostate cancer: Extended, limited, or not at all? Curr. Opin. Urol. 20, 211-217 (2010).

2. Fujimoto, N., Shiota, M., Tomisaki, I., Minato, A. \& Yahara, K. Reconsideration on clinical benefit of pelvic lymph node dissection during radical prostatectomy for clinically localized prostate cancer. Urol. Int. 103, 125-136 (2019).

3. Bader, P., Burkhard, F. C., Markwalder, R. \& Studer, U. E. Disease progression and survival of patients with positive lymph nodes after radical prostatectomy. Is there a chance of cure? J. Urol. 169, 849-854 (2003). 
4. Fossati, N. et al. Identifying the Optimal Candidate for Salvage Lymph Node Dissection for Nodal Recurrence of Prostate Cancer: Results from a Large, Multi-institutional Analysis. Eur. Urol. 75, 176-183 (2019).

5. Heidenreich, A., Varga, Z. \& Von Knobloch, R. Extended pelvic lymphadenectomy in patients undergoing radical prostatectomy: High incidence of lymph node metastasis. J. Urol. 167, 1681-1686 (2002).

6. Allaf, M. E., Palapattu, G. S., Trock, B. J., Carter, H. B. \& Walsh, P. C. Anatomical extent of lymph node dissection: Impact on men with clinically localized prostate cancer. J. Urol. 172, 1840-1844 (2004).

7. Touijer, K. et al. Standard Versus Limited Pelvic Lymph Node Dissection for Prostate Cancer in Patients With a Predicted Probability of Nodal Metastasis Greater Than 1\%. J. Urol. 178, 120-124 (2007).

8. Lestingi, J. F. P. et al. Extended Versus Limited Pelvic Lymph Node Dissection During Radical Prostatectomy for Intermediate- and High-risk Prostate Cancer: Early Oncological Outcomes from a Randomized Phase 3 Trial. Eur. Urol. 79, 595-604 (2021).

9. Touijer, K. A. et al. Limited versus Extended Pelvic Lymph Node Dissection for Prostate Cancer: A Randomized Clinical Trial. Eur. Urol. Oncol. 1-8 (2021) doi:10.1016/j.euo.2021.03.006.

10. Koie, T. et al. Safety and effectiveness of neoadjuvant luteinizing hormone-releasing hormone agonist plus low-dose estramustine phosphate in high-risk prostate cancer: A prospective single-arm study. Prostate Cancer Prostatic Dis. 15, 397-401 (2012).

11. Koie, T. et al. Neoadjuvant luteinizing-hormone-releasing hormone agonist plus low-dose estramustine phosphate improves prostate-specific antigen-free survival in high-risk prostate cancer patients: a propensity score-matched analysis. Int. J. Clin. Oncol. 20, 1018-1025 (2015).

12. Tsukamoto, S. et al. Long-term follow-up of the randomized trial of mesorectal excision with or without lateral lymph node dissection in rectal cancer (JCOG0212). Br. J. Surg. 107, 586-594 (2020).

13. Gschwend, J. E. et al. Extended Versus Limited Lymph Node Dissection in Bladder Cancer Patients Undergoing Radical Cystectomy: Survival Results from a Prospective, Randomized Trial(Figure presented.). Eur. Urol. 75, 604-611 (2019).

14. Hulscher, J. B. F. et al. Extended Transthoracic Resection Compared with Limited Transhiatal Resection for Adenocarcinoma of the Esophagus. N. Engl. J. Med. 347, 1662-1669 (2002).

15. Onenkamp, J. J. B., Ermans, J. H. \& Asako, M. S. The Ne w E $n \mathrm{~g} I$ a nd Jo u $r \mathrm{n}$ a I of Me d ic i ne EXTENDED LYMPH-NODE DISSECTION FOR GASTRIC CANCER. (1999).

16. Riall, T. S. et al. Pancreaticoduodenectomy with or without distal gastrectomy and extended retroperitoneal lymphadenectomy for periampullary adenocarcinoma - Part 3: Update on 5-year survival. 
J. Gastrointest. Surg. 9, 1191-1206 (2005).

17. Izbicki, J. R. et al. Effectiveness of radical systematic mediastinal lymphadenectomy in patients with resectable non-small cell lung cancer results of a prospective randomized trial. Ann. Surg. 227, 138144 (1998).

18. Fisher, B. et al. Twenty-Five-Year Follow-up of a Randomized Trial Comparing Radical Mastectomy, Total Mastectomy, and Total Mastectomy Followed by Irradiation. N. Engl. J. Med. 347, 567-575 (2002).

19. Harter, P. et al. A Randomized Trial of Lymphadenectomy in Patients with Advanced Ovarian Neoplasms. N. Engl. J. Med. 380, 822-832 (2019).

20. Mottet, N. et al. EAU-EANM-ESTRO-ESUR-SIOG Guidelines on Prostate Cancer-2020 Update. Part 1: Screening, Diagnosis, and Local Treatment with Curative Intent. Eur. Urol. 79, 243-262 (2021).

21. Sanda, M. G. et al. Clinically Localized Prostate Cancer: AUA/ASTRO/SUO Guideline. Part II: Recommended Approaches and Details of Specific Care Options. J. Urol. 199, 990-997 (2018).

22. Fossati, N. et al. The Benefits and Harms of Different Extents of Lymph Node Dissection During Radical Prostatectomy for Prostate Cancer: A Systematic Review. Eur. Urol. 72, 84-109 (2017).

23. Suardi, N. et al. Indication for and extension of pelvic lymph node dissection during robot-assisted radical prostatectomy: An analysis of five European institutions. Eur. Urol. 66, 635-643 (2014).

24. Narita, T. et al. The impact of extended lymph node dissection versus neoadjuvant therapy with limited lymph node dissection on biochemical recurrence in high-risk prostate cancer patients treated with radical prostatectomy: a multi-institutional analysis. Med. Oncol. 34, 1-6 (2017).

25. Jung, J. H. et al. Extended pelvic lymph node dissection including internal iliac packet should be performed during robot-assisted laparoscopic radical prostatectomy for high-risk prostate cancer. $J$. Laparoendosc. Adv. Surg. Tech. 22, 785-790 (2012).

26. Moris, L. et al. Impact of Lymph Node Burden on Survival of High-risk Prostate Cancer Patients Following Radical Prostatectomy and Pelvic Lymph Node Dissection. Front. Surg. 3, 1-9 (2016).

27. Gandaglia, G. et al. Robot-assisted Radical Prostatectomy and Extended Pelvic Lymph Node Dissection in Patients with Locally-advanced Prostate Cancer. Eur. Urol. 71, 249-256 (2017).

28. Porcaro, A. B. et al. Clinical Factors Predicting Bilateral Lymph Node Invasion in High-Risk Prostate Cancer. Urol. Int. 99, 392-399 (2017).

29. Zhang, L. et al. The Impact of Neoadjuvant Hormone Therapy on Surgical and Oncological Outcomes for Patients With Prostate Cancer Before Radical Prostatectomy: A Systematic Review and Meta-Analysis. Front. Oncol. 10, (2021). 
30. Kumar, S. et al. Neo-adjuvant and adjuvant hormone therapy for localised and locally advanced prostate cancer. Cochrane Database Syst. Rev. (2006) doi:10.1002/14651858.CD006019.pub2.

31. Briganti, A. et al. Updated nomogram predicting lymph node invasion in patients with prostate cancer undergoing extended pelvic lymph node dissection: The essential importance of percentage of positive cores. Eur. Urol. 61, 480-487 (2012).

32. Iwamura, H. et al. Evaluation of Tumor Viability for Primary and Bone Metastases in Metastatic Castration-Resistant Prostate Cancer Using Whole-Body Magnetic Resonance Imaging. Case Rep. Urol. 2018, 1-7 (2018).

33. Johnston, E. W. et al. Multiparametric whole-body 3.0-T MRI in newly diagnosed intermediate- and high-risk prostate cancer: diagnostic accuracy and interobserver agreement for nodal and metastatic staging. Eur. Radiol. 29, 3159-3169 (2019).

34. Hofman, M. S. et al. Prostate-specific membrane antigen PET-CT in patients with high-risk prostate cancer before curative-intent surgery or radiotherapy (proPSMA): a prospective, randomised, multicentre study. Lancet 395, 1208-1216 (2020).

35. Tu, X. et al. The Role of 68Ga-PSMA Positron Emission Tomography/Computerized Tomography for Preoperative Lymph Node Staging in Intermediate/High Risk Patients With Prostate Cancer: A Diagnostic Meta-Analysis. Front. Oncol. 10, 1-11 (2020).

36. Paradis, C. Bias in surgical research. Ann. Surg. 248, 180-188 (2008).

37. Paner, G. P. et al. Updates in the Eighth Edition of the Tumor-Node-Metastasis Staging Classification for Urologic Cancers. Eur. Urol. 73, 560-569 (2018).

38. D'Amico, A. V. et al. Biochemical outcome after radical prostatectomy, external beam radiation therapy, or interstitial radiation therapy for clinically localized prostate cancer. J. Am. Med. Assoc. 280, 969-974 (1998).

39. Dindo, D., Demartines, N. \& Clavien, P. A. Classification of surgical complications: A new proposal with evaluation in a cohort of 6336 patients and results of a survey. Ann. Surg. 240, 205-213 (2004).

40. Epstein, J. I. et al. The 2005 International Society of Urological Pathology (ISUP) consensus conference on Gleason grading of prostatic carcinoma. Am. J. Surg. Pathol. 29, 1228-1242 (2005).

41. Epstein, J. I. et al. The 2014 international society of urological pathology (ISUP) consensus conference on gleason grading of prostatic carcinoma definition of grading patterns and proposal for a new grading system. Am. J. Surg. Pathol. 40, 244-252 (2016).

42. Austin, P. C. \& Stuart, E. A. Moving towards best practice when using inverse probability of treatment weighting (IPTW) using the propensity score to estimate causal treatment effects in 
observational studies. Stat. Med. 34, 3661-3679 (2015).

\section{Tables}

Table 1. Baseline characteristics.

\begin{tabular}{|c|c|c|c|c|}
\hline & $\begin{array}{l}\text { Overall } \\
(n=516)\end{array}$ & $\begin{array}{l}\text { Limited-PLND } \\
\text { group } \\
(\mathrm{n}=283)\end{array}$ & $\begin{array}{l}\text { Non-PLND } \\
\text { group } \\
(n=233)\end{array}$ & $\begin{array}{l}P- \\
\text { value }\end{array}$ \\
\hline Median age, yr (IQR) & $\begin{array}{l}68(65- \\
72)\end{array}$ & $68(64-72)$ & $69(66-72)$ & 0.040 \\
\hline$E C O G-P S \geq 1, n(\%)$ & $2(0.4)$ & $1(0.4)$ & $1(0.4)$ & 1.000 \\
\hline Anticoagulant use, $\mathrm{n}(\%)$ & $33(6.4)$ & $23(8.1)$ & $10(4.3)$ & 0.103 \\
\hline Median initial PSA, ng/mL (IQR) & $\begin{array}{l}9.3(6.1- \\
17.1)\end{array}$ & $9.1(6.0-16.6)$ & $\begin{array}{l}9.4(6.3- \\
17.2)\end{array}$ & 0.698 \\
\hline Biopsy Gleason score, n (\%) & & & & $<0.001$ \\
\hline 6 (3+3; ISUP GG1) & $13(2.5)$ & $3(1.1)$ & $10(4.3)$ & \\
\hline 7 (3+4; ISUP GG2) & $70(13.6)$ & $30(10.6)$ & $40(17.2)$ & \\
\hline 7 (4+3; ISUP GG3) & $40(7.8)$ & $14(4.9)$ & $26(11.2)$ & \\
\hline $8(4+4,3+5,5+3 ;$ ISUP GG4) & $128(24.8)$ & $77(27.2)$ & $51(21.9)$ & \\
\hline $9,10(4+5,5+4,5+5 ;$ ISUP GG5) & $265(51.4)$ & $159(56.2)$ & $106(45.5)$ & 0.022 \\
\hline Clinical tumor stage, n (\%) & & & & $<0.001$ \\
\hline cT1 & $151(29.3)$ & $98(34.6)$ & $53(22.7)$ & \\
\hline cT2 & $154(29.8)$ & $98(34.6)$ & $56(24.0)$ & \\
\hline cT3 & $211(40.9)$ & $87(30.7)$ & $124(53.2)$ & $<0.001$ \\
\hline $\begin{array}{l}\text { Median risk of LNI (Briganti nomogram), } \\
\% \text { (IQR) }\end{array}$ & $16(8-37)$ & $14(7-32)$ & $18(8-46)$ & 0.024 \\
\hline Risk of $\mathrm{LNI}<5 \%, \mathrm{n}(\%)$ & $43(8.3)$ & $27(9.5)$ & $16(6.9)$ & 0.337 \\
\hline Neoadjuvant therapy, n (\%) & $\begin{array}{l}516 \\
(100.0)\end{array}$ & $283(100.0)$ & $233(100.0)$ & 1.000 \\
\hline $\begin{array}{l}\text { Median duration of neoadjuvant therapy, } \\
\text { months (IQR) }\end{array}$ & $\begin{array}{l}8.3(7.0- \\
9.9)\end{array}$ & $8.1(7.0-9.4)$ & $\begin{array}{l}8.5(7.1- \\
10.2)\end{array}$ & 0.018 \\
\hline
\end{tabular}

PLND; pelvic lymph node dissection, IQR; interquartile range, ECOG-PS; Eastern Cooperative Oncology Group Performance Status, PSA; prostate-specific antigen, ISUP GG; the International Society of 
Urological Pathology grade group, LNl; lymph node invasion

Table 2. Surgical and pathological outcomes.

Page 14/19 


$\begin{array}{llll}\begin{array}{l}\text { Overall } \\ (n=516)\end{array} & \text { Limited-PLND } & \begin{array}{l}\text { Non-PLND } \\ \text { group }\end{array} & \begin{array}{l}P \text { - } \\ \text { value }\end{array} \\ & (n=283) & (n=233) & \end{array}$

Surgery type, n (\%)

\begin{tabular}{|c|c|c|c|c|}
\hline RARP & $379(73.4)$ & $152(53.7)$ & $227(97.4)$ & \\
\hline RRP & $137(26.6)$ & $131(46.3)$ & $6(2.6)$ & \\
\hline Median operation time, min (IQR) & $\begin{array}{l}156(131- \\
184)\end{array}$ & $152(121-182)$ & $\begin{array}{l}161(138- \\
189)\end{array}$ & 0.001 \\
\hline Median blood loss, mL (IQR) & $50(20-243)$ & $100(25-735)$ & $25(10-50)$ & $<0.001$ \\
\hline Prostatectomy Gleason score, n (\%) & & & & $<0.001$ \\
\hline no residual tumor & $55(10.7)$ & $29(10.2)$ & $26(11.2)$ & \\
\hline $6(3+3 ;$ ISUP GG1) & $3(0.6)$ & $1(0.4)$ & $2(0.9)$ & \\
\hline 7 (3+4; ISUP GG2) & $12(2.3)$ & $1(0.4)$ & $11(4.7)$ & \\
\hline 7 (4+3; ISUP GG3) & $63(12.2)$ & $11(3.9)$ & $52(22.3)$ & \\
\hline $8(4+4,3+5,5+3 ;$ ISUP GG4) & $21(4.1)$ & $19(6.7)$ & $2(0.9)$ & \\
\hline $9,10(4+5,5+4,5+5 ;$ ISUP GG5) & $362(70.2)$ & $222(78.4)$ & $140(60.1)$ & $<0.001$ \\
\hline Pathological tumor stage, n (\%) & & & & 0.584 \\
\hline урт0 & $55(10.7)$ & $29(10.2)$ & $26(11.2)$ & \\
\hline урт2 & $314(60.9)$ & $168(59.4)$ & $146(62.7)$ & \\
\hline урТ3 & $147(28.5)$ & $86(30.4)$ & $61(26.2)$ & 0.327 \\
\hline Surgical margin positive, n (\%) & $44(8.5)$ & $17(6.0)$ & $27(11.6)$ & 0.027 \\
\hline $\begin{array}{l}\text { Median number of dissected nodes, } \\
n(I Q R)\end{array}$ & & $4(3-7)$ & & \\
\hline
\end{tabular}

Number of positive nodes, $\mathrm{n}(\%)$

\begin{tabular}{|c|c|c|c|c|}
\hline 0 & & $281(99.2)$ & & \\
\hline 1 & & $1(0.4)$ & & \\
\hline 2 & & $1(0.4)$ & & \\
\hline Biochemical recurrence, $\mathrm{n}(\%)$ & $85(16.5)$ & $55(19.4)$ & $30(12.9)$ & \\
\hline Cancer-specific mortality, n (\%) & $5(1.0)$ & $4(1.4)$ & $1(0.4)$ & \\
\hline All-cause mortality, n (\%) & $13(2.5)$ & $10(3.5)$ & $3(1.3)$ & \\
\hline Median follow-up periods, months & $57(31-81)$ & $77(63-94)$ & $34(20-46)$ & $<0.001$ \\
\hline
\end{tabular}


PLND; pelvic lymph node dissection, RARP; robot-assisted radical prostatectomy, RRP; retropubic radical prostatectomy, IQR; interquartile range, ISUP GG; the International Society of Urological Pathology grade group

Table 3. Multivariate Cox regression analysis for biochemical recurrence-free survival in the overall cohort.

\begin{tabular}{llll} 
Factor & & HR $(95 \% \mathrm{Cl})$ & $P$-value \\
\hline Age & Continuous & $0.95(0.92-0.99)$ & 0.013 \\
\hline Initial PSA & Continuous & $1.02(1.01-1.03)$ & 0.001 \\
\hline Biopsy Gleason score & ISUP GG1-5 & $1.18(0.95-1.46)$ & 0.130 \\
\hline Surgery type & RARP vs RRP & $0.98(0.74-1.29)$ & 0.870 \\
\hline Pathological tumor stage & ypT0-3 & $2.49(1.61-3.85)$ & $<0.001$ \\
\hline Surgical margin & Positive & $1.94(1.08-3.49)$ & 0.026 \\
\hline PLND & Limited PLND vs no PLND & $0.67(0.38-1.18)$ & 0.160
\end{tabular}

HR; hazard ratio, Cl; confidence interval, PSA; prostate-specific antigen, ISUP GG; the International Society of Urological Pathology grade group, RARP; robot-assisted radical prostatectomy, RRP; retropubic radical prostatectomy, PLND; pelvic lymph node dissection

\section{Figures}


Figure 1
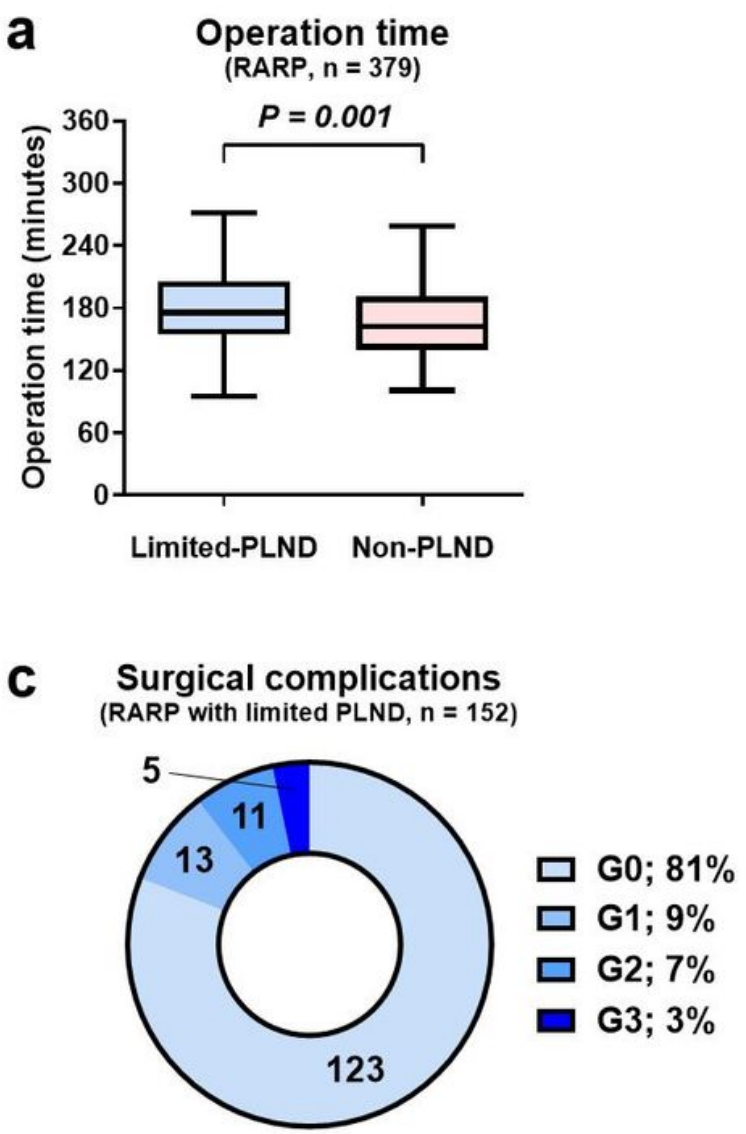

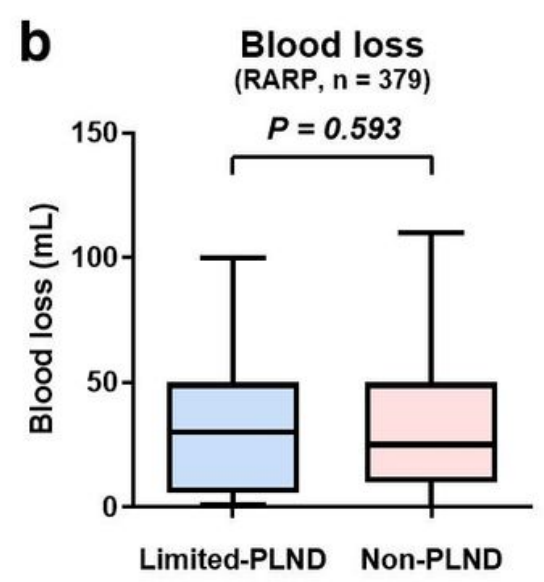

d Surgical complications

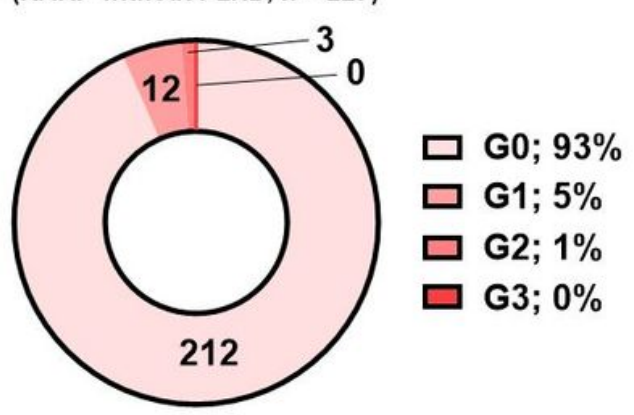

Figure 1

Operation time (a), blood loss (b), and surgical complications (c, d) between the limited-PLND and nonPLND groups (excluding RRP cases). PLND; pelvic lymph node dissection, RARP; robot-assisted radical prostatectomy, RRP; retropubic radical prostatectomy 
Figure 2

a

Age at surgery

(Limited-PLND group, $n=283$ )

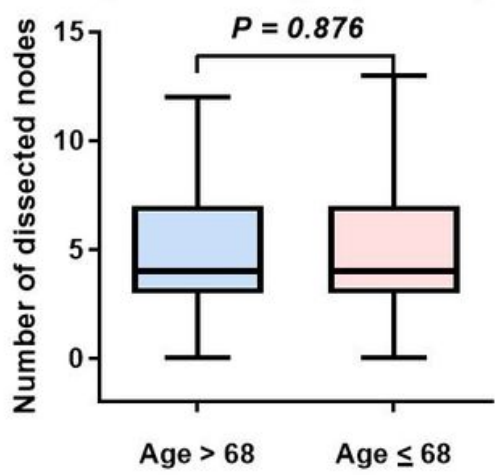

C

Surgery type

(Limited-PLND group, $n=283$ )

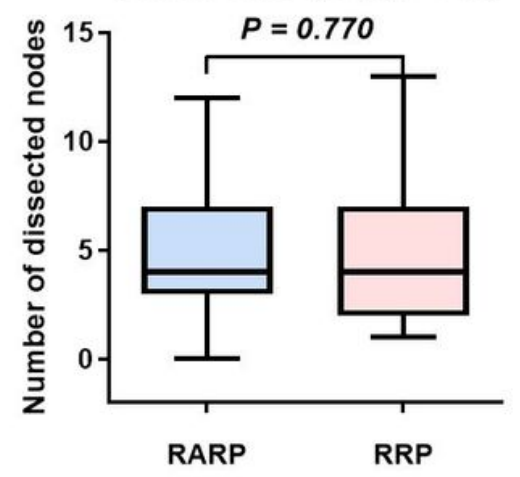

d

Clinical tumor stage

(Limited PLND group, $\mathrm{n}=283$ )

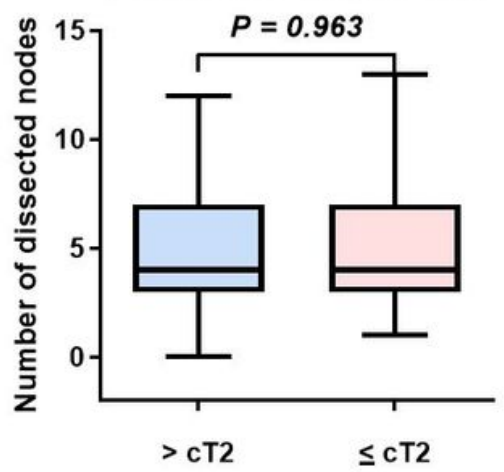

Anticoagulant use

(Limited-PLND group, $\mathrm{n}=\mathbf{2 8 3}$ )

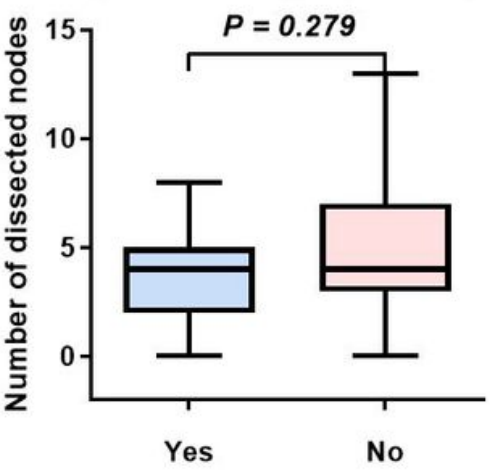

e Biopsy Gleason score

(Limited PLND group, $\mathrm{n}=283$ )

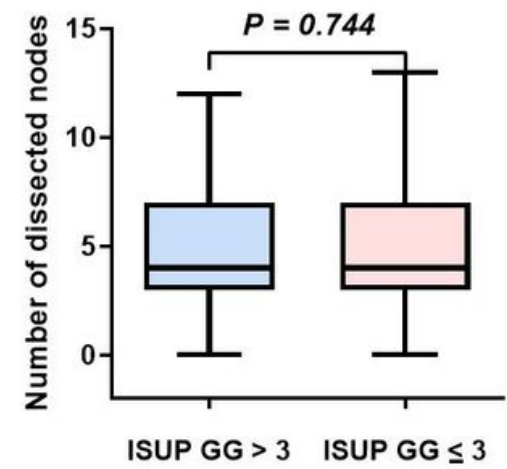

Figure 2

Number of dissected nodes stratified by age at surgery (a), anticoagulant use (b), surgery type (c), clinical tumor stage (d), and biopsy Gleason score (e) in the limited-PLND group. PLND; pelvic lymph node dissection, ISUP GG; the International Society of Urological Pathology grade group 
Figure 3

Biochemical recurrence-free survival (unadjusted)

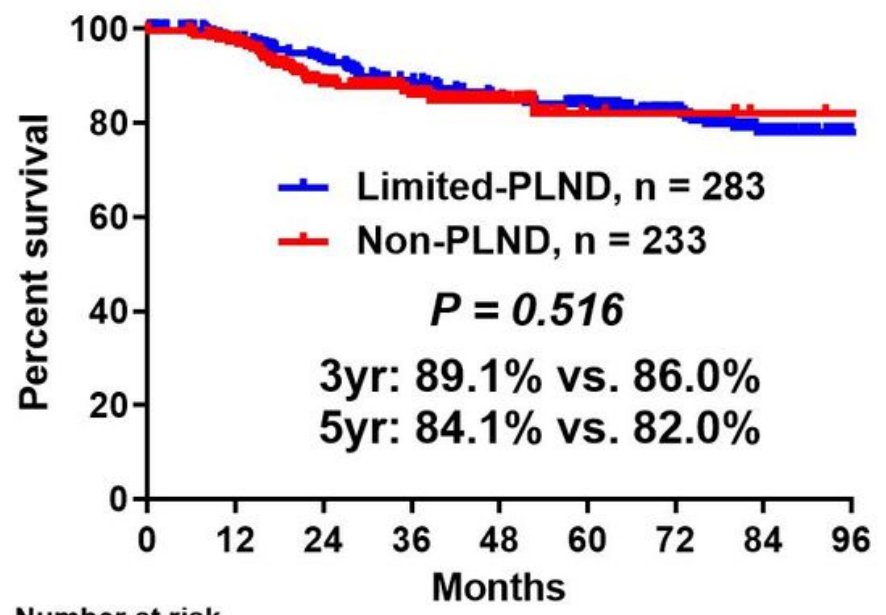

Number at risk

$\begin{array}{ccccccccc}1283 & 247 & 232 & 215 & 204 & 189 & 136 & 80 & 43 \\ -233 & 207 & 141 & 95 & 47 & 7 & 6 & 3 & 2\end{array}$

b Biochemical recurrence-free survival

(Age, initial PSA, biopsy Gleason score, surgery type, pathological tumor stage, and surgical margin-adjusted model)

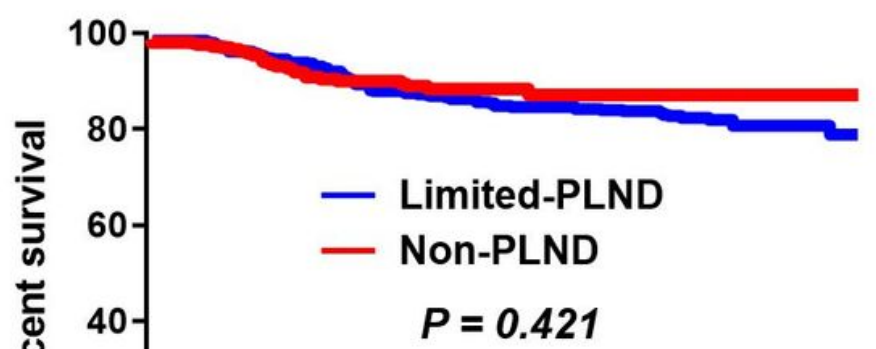

HR 1.31 (0.68-2.52)

\section{Figure 3}

Unadjusted (a) and IPTW-adjusted biochemical recurrence-free survival (b) between the limited-PLND and non-PLND groups. IPTW; inverse probability of treatment weighting, PLND; pelvic lymph node dissection, PSA; prostate-specific antigen

\section{Supplementary Files}

This is a list of supplementary files associated with this preprint. Click to download.

- SciRepsuppletable.docx 